\title{
Téoros
}

Revue de recherche en tourisme

\section{Le « capital humain ». S.O.S. compétences dispersées}

\section{Sylvie Gagnon et Jean Pelletier}

Volume 8, numéro 1, mars 1989

France-Québec

URI : https://id.erudit.org/iderudit/1080359ar

DOI : https://doi.org/10.7202/1080359ar

Aller au sommaire du numéro

Éditeur(s)

Université du Québec à Montréal

ISSN

0712-8657 (imprimé)

1923-2705 (numérique)

Découvrir la revue

Citer cet article

Gagnon, S. \& Pelletier, J. (1989). Le " capital humain ». S.O.S. compétences dispersées. Téoros, 8(1), 34-36. https://doi.org/10.7202/1080359ar d'utilisation que vous pouvez consulter en ligne.

https://apropos.erudit.org/fr/usagers/politique-dutilisation/ 


\section{Le "capital humain" S.0.S. compétences dispersées}

Nombre d'intervenants publics et privés québécois commencent à prendre conscience que le tourisme mérite d'être pris au sérieux comme facteur de développement socioeconomique tant en termes d'impacts que de retombées. Mais, malgré plusieurs investissements, programmes de soutien et mesures réglementaires depuis 15 ans, il est encore prématuré d'affirmer que les enjeux sont pris correctement en compte.

Depuis la Conférence socio-économique sur le tourisme tenue à Sherbrooke en $1978^{(1)}$, plusieurs versions de plusieurs documents de travail ont été préparés: les objectifs collectifs du développement demeurent cependant des denrées rares sauf en ce qui concerne la stratégic marketing pour les 36 prochains mois (voir à ce sujet le texte de Suzanne Chassé dans le présent numéro). Dans ce contexte, les themes relatifs aux ressources humaines (formation et recyclage) soulevvent quelques interrogations fort préoccupantes. Il devient urgent de mettre fin au cafouillage et d'impliquer l'ensemble des partenaires pour convenir de lignes directrices et de priorités d'action.

Faute de donnes comparatives, il est difficile de dire si le Québec fait plus et mieux que les autres pays en matiere de formation académique dans le champ récréo-touristique. Force est de constater cependant que le tourisme est devenu ici un mot à la mode. Alors qu'il y a à peine 20 ans la formation dans ce domaine était pratiquement inexistante, on a rattrapé le temps "perdu" depuis une douzaine d'années. On dénombre actuellement un très grand nombre d'institutions offrant des centaines de cours et programmes. On pourrait présumer que cette effervescence est liée aux besoins du marché du travail mais ce serait aller un peu trop vite en termes d'appréciation. C'est un secret de polichinelle que, dans le domaine du tourisme, formateurs et employeurs ont de la difficulté à travailler ensemble pour une meilleure adéquation de l'offre et de la demande de ressources humaines.

Les plus récentes décisions mises de l'avant par les responsables du ministere de l'Education du Québec (MEQ) en ce qui concerne la formation des agents de voyages l'ont eté sans aucune concertation avec le ministère du Tourisme et les collèges d'enseignement général et professionnel (CÉGEP) qui forment les techniciens en tourisme, et sans consultation avec l'organisme qui regroupe et représente les agences (ACTA-Québec).

\section{Le futur au présent}

Un nombre grandissant de leaders d'opinion parlent de plus en plus des industries du tourisme et du woyage comme d'un domaine où l'avenir est rempli de bonnes nouvelles: plus d'un sont en train d'épouser la thèse que ces industries s"inscriront au premier rang économique au tournant du siècle. Bien malin qui peut prévoir à court terme tous les défis de la prochaine décennie.

Les tendances sont difficiles à observer quand on se place dans une perspective à moyen et long termes. Dans 10 ou 20 ans, quels seront les comportements des gens d'affaires, des congressistes et des vacanciers? Quelles seront les destinations privilégiées? Il est certain (ou presque!) que des segments de marché sous-estimés vont émerger pendant que de nouveaux pays, régions ou villes vont investir massivement dans la promotion et présenter des propositions alléchantes. Comme un nombre de plus en plus important de pays jugent que le tourisme est une source appréciable de recettes et de retombes économiques (emplois inclus), il faut s'attendre à voir une concurrence accrue, voire féroce, tant à l'échelle internationale (150 pays intéressés) qu"à l'échelle nationale ( 50 états américains ou 10 provinocs canadiennes) et régionale ("countys" du côté américain, régions du côté québécois).

L'accroissement du mouvement des personnes attendu dans le monde d'ici l'an 2000 amène à penser que seuls les pays qui auront su contrôler ou s'insérer dans des réseaux, y imposer leurs normes de transaction afin d'y faire circuler leurs produits, seront les gagnants de cette compétition. Les voyageurs de l'an 1999 ... ou 2009 nous sont encore trop mal connus mais plusieurs facteurs peuvent nous éveiller: vieillissement des populations des pays dits développés, émergence de destinations alternatives de découverte dans tous les coins de la planète, déplacements éventuellement massifs de visiteurs de pays qui finiront par dégager des nouvelles sources de richesse collective.

Dans I'hypothèse plausible qu'il y ait, malgré une récession ou deux, un minimum de croissance conomique au cours des dix prochaines années, la population devrait disposer d'un revenu discrétionnaire plus elevé. Cela est prévisible autant au Québec qu'au Canada, aux États-Unis ou en Europe. Qui plus est, l'Accord de libre-echange canadoanéricain et les nouvelles règles de l'Europe 
post 1992 vont favoriser la mobilité des capitaux et des personnes. Les conditions sont donc en place pour un accroissement de la demande de produits récréo-touristiques ou plutôt pour la poursuite des tendances évolutives constatées depuis l'après-guerre.

Il est certain que plusieurs secteurs profiteront plus que d'autres de la hausse de cette demande. De la méme manière, certains pays en bénéficieront aussi plus que d'autres. II est même fort possible, dans ce contexte général, que certains secteurs d'activités ou certains pays subissent des reculs significatifs.

Rien n'est assuré dans ce domaine. Tout dépend des types de consommation récréotouristiques qui seront privilégiés et de la concurrence que ne manquent pas de se livrer tous et chacun afin de $s^{7}$ accaparer une plus grande part des marchés. Une des conditions essentielles du succès et de la réussite repose sur la qualité des ressources humaines. Et le Québec, comme les autres, est en train de vivre une mutation profonde sur le plan du marché du travail. Qui fera quoi? Avec quel bagage de connaissances? Avec quels outils?

Lors de la Conférence nationale du tourisme de demain tenue à Ottawa en octobre 1985 , un document de travail soulignait que: "I'industrie doit travailler en étroite collaboration avec les maisons d'enseignement et le gouvernement afin de s'assurer que le système d'éducation (leur) dispense des connaissances approprićes. Les maisons d'enseignement doivent également s'assurer d'offrir la formation à toutes les entreprises et (leurs) travailleurs qui veulent améliorer leurs connaissances".,"(2) Avons-nous suffisamment été sensibilisés à ce qui se fait déjà?

\section{La prolifération des cours}

A la fin des années 70 , il était relativement facile de cerner la question de la formation en tourisme au Québec: un programme de niveau collégial offert par quatre institutions (CEGEP de Granby, de Matane, Collège LaSalle et Institut de tourisme et d'hötellerie du ministère du Tourisme du Québec), un autre de niveau universitaire offert par 1'Université du Québec à Montréal (UQAM) et la formation d'agents de voyages dispensée par une institution privée créé par des gens du milieu (École April-Fortier).

Aujourd'hui, il n'en va plus de même. Avant de formuler quelques réflexions sur l'état de la situation, se pose (et scrieusement!) le problème du dénombrement des différents cours et programmes offerts et, par le fait même, du nombre de diplômés (tous diplômes confondus...). Il n'est plus une région, plus une institution qui n'affiche de formation en tourisme: écoles publiques ou privées de tous niveaux, à temps plein ou à temps partiel, à la leçon ou de façon intensive; diplômes du MEQ, attestations, certificats ou reconnaissance par des associations; pour les jeunes et les adultes; avec ou sans pré-requis; en gestion, en marketing, en accueil, en "agences de voyages", etc...

C'est ce travail de recensement qui a ćté entrepris, à l'été 1984, dans le cadre d'une étude sur la formation et l'emploi en tourisme $^{3\rangle}$. Résultat: " ... 538 cours et programmes en tourisme (loisir, hôtellerie, etc..) sont dispensés dans 171 institutions privées et publiques au Québec"t(4); “... 10000 personnes sont formées pour une industrie qui compte 137000 emplois et présente un taux de croissance annuel de 6\%"

Bien que cette recherche soit concluante quant à la nécessité de contrôler la prolifération, nous pouvons constater aujourd'hui que l'appel au calme n'a pas été entendu. En effet, cinq ans plus tard, meme en excluant les secteurs du loisir, de l'hôtellerie et de la restauration, d'autres intervenants se sont ajoutés au tableau et d'autres actions pointent à I'horizon:

1- Deux autres collèges (Mérici et Champlain) offrent maintenant le programme Techniques touristiques;

2- Des CÉGEP offrent des cours menant à une attestation d'études collégiales (AEC). Le contenu de ces cours ainsi que leur durée sont laissés à la discrétion des établissements et aucun pré-requis n'est nécessaire pour s'y inscrire. Actuellement, plus d'une demi-douzaine de CÉGEP offrent des AEC en tourisme: initiation à l'administration touristique et conseiller en tourisme extérieur (c'est-àdire agent de voyages!);

3- Le collège LaSalle a mis sur pied des ateliers du tourisme qui s'adressent spécifiquement aux intervenants du voyage. Il s'agit de quatre programmes distincts (gestion, destinations, automatisation et guide réceptif) auxquels les gens peuvent s'inscrire à la pièce ou en "achetant" le programme au complet. Il est à noter que de plus en plus d'institutions scolaires et d'organismes privés offrent ce genre de cours ou séminaires spécialisés qui, sans mener à un diplöme officiel, doivent tout de même répondre à certains besoins du marché;

4- Le ministère de l'Éducation du Québec, dans le cadre de sa réforme de l'enseignement professionnel au niveau secondaire, en est à faire approuver par le Conseil supérieur de l'Education un programme de 900 heures visant la formation d'agents de voyages. Fort vraisemblablement, ce programme sera offert, dès l'automne prochain, par au moins cing ou six 6coles polyvalentes et par le Collège de secrétariat moderne (établissement privé). Dans le sillage de l'intégration jeunesadultes à la formation dite régulière, ce programme remplacera les cours d'agents de voyages offerts dans le cadre de l'éducation aux adultes;
5- Le module de gestion et intervention touristiques de l'université du Québec à Montréal et d'autres milieux universitaires travaillent depuis deux ans à la mise sur pied d'un projet de maitrise;

6- L'Institut de Tourisme et d'Hôtellerie du Québec (ITHQ), associé à un établissement universitaire français (Toulouse), a débuté à l'automne 87 une maîtrise des Sciences et Techniques (MST) offrant deux options: gestion hôtellerie/restauration et gestion tourisme. Cette maitrise, loin d'avoir fait l'unanimité, ne serait plus offerte à compter de l'automne prochain. Il importe de signaler que l'ITHQ est devenu récemment un organisme parapublic ne relevant plus directement du ministère du Tourisme du Québec;

7- Un projet montréalais d'Institut international de formation en tourisme fait présentement l'objet d'une étude de faisabilité. Rappelons qu'un Institut international de formation en gestion aéronautique civile a êté créé à Montréal en 1987.

La multiplication des diplômés À ce chapitre, le constat de l'étude sur la formation et l'emploi déjà cité ne peut être qu'amplifié. Les quelques faits rapportés cidessus n'annoncent pas une meilleure adéquation sur le plan quantitatif entre la maind'oeuvre formée et les besoins du marché du travail.

Si le nombre total de diplômés des programmes réguliers de niveaux collégial et universitaire demeure relativement stable - la baisse des uns étant compensée par l'augmentation des autres - c'est du côté des autres filières que la surenchère se fait sentir.

Limitons-nous à l'exemple des agents de voyages pour lesquels des donnees, quant aux besoins futurs, existent ${ }^{(6)}$. Le Système des projections des professions au Canada (SPPC) évalue à 981 la demande d'agents de voyages au Québec pour la période $1986-1995^{(7)}$, ce qui donne une moyenne annuelle de 109 nouveaux emplois d'ici 1995. Il est évident qu'au total, les écoles privées spécialisées (April-Fortier, Publiwox, etc...), les CÉGEP (AEC en tourisme extérieur et près de $40 \%$ des techniciens ${ }^{(2)}$ ) et les polyvalentes (à compter de septembre prochain) formeront annuellement beaucoup plus que 109 candidats et ce sans compter les personnes qui accèderont au métier sans formation spécifique.

En plus du problème de la prolifération anarchique des programmes et des cours et de l'engouement des candidats à s'y inscrire, quels autres constats pouvons-nous tirer de la situation actuelle?

\section{Des voeux pieux}

La concertation? Tout le monde est d'accord...! Mais dans les faits, il en va tout autrement. Au-delă des besoins du marché, de lạ qualité de la formation, des problèmes de définitions ("'industries" et professions), 
de reconnaissance - de connaissance même! - des diplómés, d'autres enjeux, d'autres intérêts se présentent: les écoles publiques veulent offrir des programmes attrayants (y a-t-il des propositions plus séduisantes que les voyages?), les professeurs veulent sauvegarder leur emploi, l'ancienneté primant sur la compétence et les connaissances des enseignants; les écoles privées ont des produits à vendre (et moins de contraintes à s'ajuster à la demande); les employeurs et les intervenants ont, semble-t-il, bien d'autres chats politiques et fiscaux à fouetter...

Si les établissements de même niveau offrant le même programme collégial n"arrivent pas à se concerter, comment penser asseoir à une mẻme table deux niveaux de gouvernement, trois ministères, des établissements publics de trois niveaux - et de deux langues -, des écoles privées et des employeurs représentatifs de chaque secteur que le tourisme prétend regrouper?

Le ministère du Tourisme, premier concerné, de concert avec le MEQ et le ministère de l'Enseignement supéricur et de la Science (MESS), commence à promettre de s'intéresser à la formation et au perfectionnement de la main-d'oeuvre touristique. l'ITHQ n'ćtant plus sous sa tutelle, il se doit maintenant de reconnaitre dans ses politiques et pratique l'existence des autres institutions. Jouera-t-il maintenant le rôle de leader qui lui revient à ce chapitre?

\section{Un début d'harmonisation}

La récente révision du programme universitaire de gestion et intervention touristiques donnant à celui-ci une nette orientation en gestion permet maintenant de mieux cerner les fonctions que sont appelés à remplir ces diplồnés.

Du côté gouvernemental, les travaux visant la création d'un programme de niveau secondaire pour former des agents de voyages et ceux entrepris dans le cadre de la révision du programme collégial en techniques touristiques ont amené certains responsables à s'entendre pour partager les niveaux de formation professionnelle: ${ }^{4}$...l'enseignement au niveau secondaire se préoccupe de la formation d'ouvriers spécialisés et de la maind'oeuvre dont les táches sont peu complexes et souvent répétitives. A l'enseignement collégial a été dévolu la formation professionnelle qualifiée de techniques requérant un certain niveau d'autonomie, le développement d'habiletés d'analyse et de gestion" ${ }^{n(9)}$.

Dans cette optique, on entend réaffirmer l'orientation "tourisme réceptif" des colleges tout en visant, en ce qui a trait au tourisme dit émetteur, à préparer les différents. étudiants à des fonctions auxquelles le niveau secondaire ne répondait pas. Restent les certificats universitaires, les projets de maîtrise, les écoles privées spécialisées, les cours sur mesure, les séminaires offerts par les intervenants... Si les instances officielles finissent par donner le ton, si la CCDP (actuellement en cours de révision) reconnaît les professions en tourisme, peut-être que les intéressés (formateurs et employeurs) emboîteront le pas et ce au grand profit des diplómés et travailleurs mais surtout des industries elles-mêmes.

\section{Épilogue inquiet}

La réalité québécoise n'est sürement pas unique. Le développement du tourisme repose à la fois sur une multitude de petites et moyennes entreprises qui ignorent trop souvent leur complémentarité et des entreprises nationales ou multinationales qui ont été emportées depuis quelques années dans le tourbillon de batailles financières (déréglementation, privatisation, prise de controble, regroupement, etc...) et qui ont pu faire perdre de vue les priorités en matière de qualité de service et les exigences en termes de ressources humaines.

Nous sommes à moins de 4000 jours de l'an 2000. Le Québec doit dès maintenant se tracer des objectifs et se donner des moyens d'être encore dans la course aux côtés des plus performants. Dans les conditions actuelles, ce ne sera pas une mince tâche: $y$ aura$t$-il suffisamment d'hommes et de femmes volontaires et capables de cerner de façon continue les besoins et les exigences qui en découlent et de relever le défi de l'excellence? Souhaitons-le

\section{Notes}

I11 Gouwernement du Québec, Le Tourisme, Perspectives de relance. Ouebec. Rapport de la Confénence sectorielle sur le tourisme, octobre-novembre 1978 , $247 \mathrm{p}$.

(2) Gouvernement du Canada, Expansion industrielle régionale, Formation et entrainement. Ottawa, 1905, $21 \mathrm{p}$.

(3) Courtade Marie-Héléne et Francoeur Manon, Tourisme: formation et emplois, Montréal, Les dossiers du CET, Centre d'Etudes du Tourisme, octobre 1994. $86 \mathrm{p}$.

(4) Ibid, P. 29

(5) Ibid, p. 57

(6) Les projections faites par les gouvernements québé. cols et canadien le sont sur la base des professions codifiées dans la Classification canadienne descriptive des professions (CCDPI. Le groupe des agents de wovages est le seul métier de l'industrie touristique qui est codifié et qui correspond précisément a un programme de formation. On ne peut etablir une telle corrélation pour les techniciens ét les bacheliers en tourisme oui ne sont pas des professions codifítes dans la CCDP. C'est d'ailleurs ce qui rend si difficile la quantification des besoins en main-d'osuvre et la définition de l'industrie en terme d'emplois.

(7) Bureau d'études socio-démographiques, Techniques du tourisme: formation et emploi. Sondege aupres des techniciens en tourisme et de leurs employeurs, Saint-Bruno de Montarville, decembre $1987, \mathrm{p} .16$

Cette enquỉtte portant sur les finissants des années 1981 à 1986 démontrent que $39,4 \%$ des techniciens en tourisme ont des emplois reliés à la vente de voyages.

(8) Belisle, Svluain et al, Les tendances professionnelles au Québec (1986-1995). Rásultats partiels. Otawa, SPPC, Emploi et immigration Canada, Direction des services 6conomiques, 1987, 109 p.

(9) Desrochers, Nora, Problématique des techniques. du tourisme, Québec. Ministére de l'Enseignement supérieur et de la science, Service des programmes, octobre 1988, p. 8. 